\title{
Respiratory Disease and Panic Attacks Among Adults in the United States*
}

\author{
Renee D. Goodwin, PhD; and Daniel S. Pine, MD
}

\begin{abstract}
Objective: To determine the association between respiratory disease and panic attacks among adults in the US population.

Method: Data were drawn from the Midlife Development in the United States Survey $(n=3,032)$, a representative sample of adults aged 25 to 74 years. Multivariate logistic regression analyses were used to determine the relationship between self-reported respiratory and other lung disease and panic attacks, major depression, generalized anxiety disorder, and alcohol/substance use disorders.

Results: After adjusting for demographic characteristics, comorbid mental disorders, and comorbid physical disorders, self-reported respiratory disease (ie, asthma, chronic bronchitis, or emphysema) was associated with a significantly increased likelihood of panic attacks (odds ratio, 1.7; confidence interval, 1.2 to 2.4 ). Other self-reported lung disease was also associated with a significantly increased odds of panic attacks (odds ratio, 2.3; confidence interval, 1.2 to 4.2), and having both self-reported respiratory disease and another lung disease was associated with increased likelihood of panic attacks (odds ratio, 4.1; confidence interval, 1.7, 9.9). These associations also persisted after adjusting for demographic characteristics, comorbid mental disorders, and physical comorbidity.

Conclusion: These findings are consistent with and extend previous clinical and epidemiologic data by showing a specific association between self-reported respiratory disease and panic attacks among adults. Future studies that investigate the relationship between respiratory disease and panic attacks, and other mental disorders, using prospectively collected data on respiratory functioning, may help to improve our understanding of the mechanism of this association.
\end{abstract}

(CHEST 2002; 122:645-650)

Key words: asthma; panic attacks; respiratory disease

Abbreviations: CIDI-SF = Composite International Diagnostic Interview Short Form; GAD = generalized anxiety disorder; MIDUS = Midlife Development in the United States

$\mathbf{P}$ revious data from clinical samples suggest that anxiety disorders, especially panic attacks, occur more often than would be expected by chance among individuals with respiratory diseases..$^{1-4}$ Anxiety and depression are common and are associated with significant functional impairment above that associated with medical morbidity alone among adult patients with COPD. ${ }^{2}$ Mood and anxiety disorders are also prevalent among both adult and pediatric patients with asthma and severe lung diseases. ${ }^{2,3}$ Anxiety and depressive symptoms are predictive of poorer asthma management, associated functional

${ }^{*}$ From the Department of Psychiatry (Dr. Goodwin), College of Physicians and Surgeons of Columbia University, Division of Epidemiology, Mailman School of Public Health, New York, NY; and the National Institute of Mental Health (Dr. Pine), Bethesda, MD.

Manuscript received August 6, 2001; revision accepted December 20, 2001.

Correspondence to: Renee D. Goodwin, PhD, 1051 Riverside Dr, Unit \#43, New York, NY 10032; e-mail: rdg66@columbia.edu

impairment, and inferior treatment outcomes among asthma patients. ${ }^{5-7}$ Moreover, though not all findings are consistent, some clinical data show a doseresponse relationship between level of anxiety and severity of airway obstruction in patients with bronchial asthma, as assessed by pulmonary function testing. ${ }^{4,8}$ The rates of respiratory illness are also higher than expected among some psychiatric patient populations, compared to the general population. ${ }^{9,10}$

As data from clinical settings are limited in their generalizability to the community since effect sizes may be skewed by selection into treatment bias, it is important to also examine this relationship using community-based data. Epidemiologic studies ${ }^{11,12}$ in adults have shown that panic disorder co-occurs with asthma more frequently than would be expected by chance. There are also data to suggest that COPD and emphysema are associated with significantly higher rates of anxiety disorders and depression in 
the community, compared to those without these disorders. ${ }^{13}$ To date, however, previous epidemiologic studies have not investigated the specificity of the relation between mental disorder and respiratory disease. If there is a unique relationship between respiratory disease and anxiety disorders or panic attacks, in contrast to other mental disorders as previous data suggest, ${ }^{11}$ then this relationship should be observable in a population-based sample. Moreover, if the association between panic attacks and respiratory disease is specific, then it should persist after adjusting for other comorbid mental disorders. This has not previously been examined.

The current study investigated the relation between self-reported respiratory disease and anxiety, depressive, and substance use disorders in a community-based sample representative of the US adult population aged 25 to 74 years. The study will first determine the relation between self-reported respiratory disease and anxiety, depressive, and substance use disorders in the population. The study will then determine the specificity of the association between respiratory disease and mental disorders. Based on results from previous clinical and epidemiologic studies, we hypothesized that respiratory disease will be associated with increased risk of panic attacks, compared with that among those without respiratory disease.

\section{Materials AND Methods}

\section{Sample}

The Midlife Development in the United States (MIDUS) Survey is a nationally representative survey of 3,032 persons aged 25 to 74 years in the noninstitutionalized civilian population of the 48 coterminous United States. ${ }^{14}$ The MIDUS Survey was carried out by the John D. and Catherine T. MacArthur Foundation Network on Successful Midlife Development between January 1995 and January 1996. The survey was based on a nationally representative random-digit-dial samples of noninstitutionalized, English-speaking adults, selected from working telephone banks in the coterminous United States. Contacted persons were informed that the survey was being carried out through Harvard Medical School, and was designed to study health and well-being during the middle years of life. They were told participation would entail a telephone interview and two mail questionnaires. When it was determined that a random respondent was appropriate for inclusion, an attempt was made to talk with this person and recruit him or her to be a participant. A study fact brochure was mailed to respondents who asked for more information before deciding, and a recontact telephone appointment was made after they received the brochure. Once random respondents decided to participate, all respondents completed a 30 -min telephone interview ( $70.0 \%$ response rate) and filled out two mailed questionnaires estimated to take $90 \mathrm{~min}$ to complete $(86.8 \%$ conditional response rate in the subsample of telephone respondents). The questionnaire mailing also included a boxed pen and a check for $\$ 20$. Data reported here were weighted to adjust for differential probabilities of selection and nonresponse. More details on the sampling procedure, field methods, and weights are available elsewhere. ${ }^{14}$

\section{Diagnostic Assessment}

The MIDUS Survey diagnoses were based on the Composite International Diagnostic Interview Short Form (CIDI-SF) scales, a series of diagnostic-specific scales that were developed from item-level analyses of the Composite International Diagnostic Interview questions in the National Comorbidity Survey. ${ }^{15,16}$ The CIDI-SF scales were designed to reproduce the full composite international diagnoses as well as possible with only a small subset of the original questions. CIDI-SF diagnoses at 12 months assessed in the MIDUS Survey included major depression, panic attacks, generalized anxiety disorder (GAD), alcohol abuse and dependence, and drug abuse and dependence (past 12-month prevalence). Panic attacks were diagnosed with the CIDI-SF. ${ }^{15}$ The sensitivity of CIDI-SF classification for panic attack is 90.0 and specificity is 99.5 ; for major depression, sensitivity is 89.6 and specificity is 93.9 ; for GAD, sensitivity is 96.6 and specificity is 99.8; for alcohol dependence, sensitivity is 93.6 and specificity is 96.2; and for substance dependence, sensitivity is 77.0 and specificity 99.9. ${ }^{17}$ Data on physical illness diagnoses were obtained through self-report. Subjects were asked whether they had ever experienced or been treated for the following physical conditions in the past 12 months: asthma, bronchitis, emphysema, other lung problems, as well as high BP/hypertension, autoimmune diseases, diabetes, ulcer, sciatica, thyroid disease, hay fever, tuberculosis, gall bladder disease, AIDS/HIV, neurologic disorders, stroke, hernia, and recurrent stomach problems. For analyses in the current study, there were three binary variables used to examine respiratory disease. One variable, termed respiratory disease, included those who endorsed having either asthma, chronic bronchitis, or emphysema. The second variable, termed lung disease, included those who endorsed having "other lung disease." The third variable, termed comorbid respiratory disease, included individuals who endorsed having both asthma, chronic bronchitis, or emphysema and other lung disease. Subjects were grouped by marital status (married, divorced/widowed, never married) and educational attainment was dichotomized into those who had and had not completed high school.

\section{Analytic Strategy}

First, differences in sociodemographic characteristics were compared between individuals with and without respiratory disease using Pearson $\chi^{2}$ tests. All tests were two sided, and significance was set at 0.05 . Second, the prevalence of major depression, panic attack, GAD, and alcohol/substance use disorders were compared between those with and without respiratory disease using the same method. Third, multiple logistic regression analyses were used to determine the relation between common mental disorders and respiratory disease, first adjusting for differences in sociodemographic characteristics and subsequently controlling for all other mental and physical morbidity.

\section{RESUlts}

\section{Description of the Sample}

The overall sample was $50.8 \%$ female, $62.3 \%$ married, $75.1 \%$ white, $5.7 \%$ black, and $2.5 \%$ Hispanic. The average respondent age was 46.35 years (SD, 13.22 years), and $60.4 \%$ of the sample completed high school. The overall response rate was $60.8 \%$. 
Self-reported respiratory or lung disease was prevalent among $13.0 \%$ of the adult population; $9.8 \%$ of the population had self-reported respiratory disease (ie, asthma, bronchitis or emphysema), 1.6\% had other lung problems, and $1.4 \%$ had both asthma, bronchitis and/or emphysema and other lung problems.

\section{Sociodemographic Characteristics Associated With Respiratory Disease}

Individuals with any self-reported respiratory or lung disease were more likely to be women and unmarried, and more likely to be separated or divorced, compared with those without self-reported respiratory or lung disease (Table 1); $75.6 \%$ of those with any respiratory disease reported asthma, and a significant majority of this group was female, although the majority of those with other lung disease and both asthma and other lung disease were men. This preponderance of women in the respiratory disease group may account for this gender difference.

\section{Relation Between Mental Disorders and Respiratory and Lung Disease}

Panic attacks and major depression were both associated with a statistically significant increase in prevalence of respiratory disease, lung disease, and comorbid respiratory and lung disease (Table 2). A similar pattern was evident in the relation between GAD and lung disease and comorbid respiratory and lung disease. A similar pattern was evident in the relation between alcohol/substance-use disorder and respiratory and lung disease, though neither GAD nor alcohol/substance use showed a significant relation with respiratory disease alone (without another lung disease). Of interest, lifetime prevalence of regular cigarette smoking was higher among respondents with both respiratory and lung disease (84.4\%), lung disease $(84.2 \%)$, and respiratory disease (73.3\%), compared with those without any respiratory disease $\left(68.7 \% ; \chi^{2}=9.4\right.$, degrees of free$\operatorname{dom}=3, \mathrm{p}=0.025)$.

\section{Adjusted Relation Between Mental Disorders and Respiratory Disease}

Respiratory disease was associated with a significantly increased likelihood of panic attacks and major depression, which persisted after adjusting for differences in sociodemographic characteristics and comorbid mental disorders (Table 3). After additionally adjusting for physical comorbidity, only the relationship between respiratory disease and panic attacks remained statistically significant.

\section{Adjusted Relation Between Mental Disorders and Other Lung Disease}

Lung disease was associated with a significantly increased odds of each of the mental disorders examined, controlling for demographic differences (Table 4). The association between lung disease and panic attacks and substance use disorders persisted after further adjustment for psychiatric comorbidity. In the final model, lung disease was only associated with a significantly increased odds of panic attack, while the relationship between lung disease and alcohol/substance use disorder ceased to be statistically significant after adjusting for physical comorbidity.

Table 1-Sociodemographic Characteristics of Adults With and Without Self-reported Respiratory Disease in the United States*

\begin{tabular}{|c|c|c|c|}
\hline $\begin{array}{l}\text { Sociodemographic } \\
\text { Characteristics }\end{array}$ & $\begin{array}{c}\text { No Respiratory } \\
\text { Disease } \\
(\mathrm{n}=2,216)\end{array}$ & $\begin{array}{c}\text { Respiratory } \\
\text { Disease } \\
(\mathrm{n}=393)\end{array}$ & $\begin{array}{c}\chi^{2}, \text { Degrees of } \\
\text { Freedom, } \\
\text { p Value }\end{array}$ \\
\hline Mean age (SD), yr & $46.8(13.1)$ & $47.5(13.1)$ & Not significant \\
\hline Gender & & & $9.8,1,0.002$ \\
\hline Male & 49.8 & 41.2 & \\
\hline Female & 50.2 & 58.8 & \\
\hline Race & & & Not significant \\
\hline White & 86.6 & 84.8 & \\
\hline Minority racial status & 13.4 & 15.2 & \\
\hline Marital status & & & $9.1,2,0.01$ \\
\hline Married & 74.0 & 66.2 & \\
\hline Separated/divorced & 19.8 & 26.0 & \\
\hline Never married & 6.3 & 7.8 & \\
\hline Education & & & Not significant \\
\hline$<11$ th grade & 38.6 & 36.9 & \\
\hline High school diploma + & 61.4 & 63.1 & \\
\hline
\end{tabular}

*Data are presented as \% unless otherwise indicated. 
Table 2-Relation Between Mental Disorders and Respiratory Disease Among Adults in the US General Population*

\begin{tabular}{|c|c|c|c|c|}
\hline Mental Disorders & $\begin{array}{l}\text { No Respiratory or } \\
\text { Lung Disease } \\
\quad(\mathrm{n}=2,235, \\
\text { reference group }) \uparrow\end{array}$ & $\begin{array}{l}\text { Respiratory } \\
\text { Disease } \\
(\mathrm{n}=343)\end{array}$ & $\begin{array}{l}\text { Lung Disease } \\
\qquad(\mathrm{n}=93)\end{array}$ & $\begin{array}{l}\text { Comorbid Respiratory } \\
\text { and Lung Disease } \\
(\mathrm{n}=43)\end{array}$ \\
\hline Panic attacks & 11.2 & $22.2+$ & $29.0_{+}^{t}$ & $41.9+$ \\
\hline Major depression & 13.1 & $21.3+$ & $24.7 \S$ & $37.2_{+}^{+}$ \\
\hline GAD & 2.5 & 3.8 & $9.6 \S$ & $11.6 \$$ \\
\hline Alcohol/substance use disorder & 2.7 & 3.2 & $7.6 \S$ & $11.6 \$$ \\
\hline
\end{tabular}

*Data are presented as \%.

$\uparrow$ Each disease group was compared to the reference group.

$+\mathrm{p}<0.001$.

$\S \mathrm{p}<0.05$.

$\| \mathrm{p}<0.01$.

\section{Adjusted Relation Between Mental Disorders and Comorbid Respiratory Disease}

Comorbid respiratory disease was associated with a significantly increased likelihood of each mental disorder examined (Table 5). This association persisted after adjusting for differences in demographic characteristics. After controlling for comorbid mental disorders, the association between panic attack, major depression, and substance use disorders and comorbid respiratory disease remained significant. After additionally controlling for physical comorbidity, only the associations between respiratory disease and panic attacks remained significant, and the relationship between GAD was again significant.

\section{DisCUSSION}

These data are consistent with and extend previous findings documenting an association between self-reported respiratory disease and increased likelihood of panic attacks among adults in the general population. This association, which persists after adjusting for differences in sociodemographic characteristics, physical illnesses, and comorbid mental disorders, is specific to panic attacks. These results also provide preliminary evidence suggesting that GAD and alcohol/substance-use disorders may also be related to respiratory and lung disease in the community.

The mechanism of the observed association between respiratory disease and panic attack remains unknown. ${ }^{18}$ Conclusions about the sequence of onset of respiratory disease and panic attack cannot be drawn from these data since they are cross-sectional and we do not have information on ages of onset. Therefore the possibility that either panic attacks lead to the onset of respiratory disease (eg, asthma) or that respiratory or lung disease lead to the development of panic attacks remain. It is also conceivable that a third factor is associated with an increased risk for the co-occurrence of respiratory disease and panic attack. Future studies that examine the relationship between outside factors or common risk factors (eg, cigarette smoking, low socioeconomic status) and the risk of the co-occurrence of respiratory disease and panic attack may prove useful in determining possible mechanisms of this association by identifying or ruling out possible routes of transmission (ie, genetic, environmental). ${ }^{19,20}$ For in-

Table 3-Relation Between Mental Disorders and Asthma/Chronic Bronchitis/Emphysema Among Adults in the US General Population*

\begin{tabular}{lcccc}
\hline \hline \multicolumn{1}{c}{ Mental Disorders } & $\begin{array}{c}\text { Unadjusted OR } \\
(95 \% \mathrm{CI})\end{array}$ & $\begin{array}{c}\text { Adjusted OR } \\
(95 \% \mathrm{CI}) \dagger\end{array}$ & $\begin{array}{c}\text { Adjusted OR } \\
(95 \% \mathrm{CI})+\end{array}$ & $\begin{array}{c}\text { Adjusted OR } \\
(95 \% \mathrm{CI}) \S\end{array}$ \\
\hline Panic attacks & $2.2(1.7,3.0) \|$ & $2.1(1.6,2.9) \|$ & $2.0(1.4,2.7) \|$ & $1.7(1.2,2.4) \|$ \\
Major depressive disorder & $1.8(1.4,2.4) \|$ & $1.7(1.3,2.3) \|$ & $1.5(1.1,2.0) \|$ & $1.1(0.8,1.6)$ \\
GAD & $1.5(0.8,2.8)$ & $1.4(0.7,2.6)$ & $0.9(0.5,1.8)$ & $0.99(0.5,2.1)$ \\
Alcohol/substance use disorder & $1.1(0.6,2.2)$ & $1.2(0.6,2.3)$ & $0.98(0.5,1.9)$ & $0.7(0.3,1.4)$ \\
\hline
\end{tabular}

$* \mathrm{OR}=$ odds ratio; $\mathrm{CI}=$ confidence interval.

† Adjusted for age, gender, race, marital status, and education.

+ Adjusted for age, gender, race, marital status, education, and comorbid mental disorders.

\$Adjusted for age, gender, race, marital status, education, comorbid mental disorders, and comorbid physical illnesses.

$\| \mathrm{p}<0.05$. 
Table 4-Relation Between Mental Disorders and Self-reported Lung Disease Among Adults in the US General Population*

\begin{tabular}{|c|c|c|c|c|}
\hline Mental Disorders & $\begin{array}{c}\text { Unadjusted } \\
\text { OR }(95 \% \text { CI })\end{array}$ & $\begin{array}{c}\text { Adjusted OR } \\
(95 \% \mathrm{CI}) \uparrow\end{array}$ & $\begin{array}{c}\text { Adjusted OR } \\
(95 \% \text { CI })_{+}^{+}\end{array}$ & $\begin{array}{c}\text { Adjusted OR } \\
(95 \% \text { CI }) \S\end{array}$ \\
\hline Panic attacks & $3.3(2.0,5.2)$ & $3.6(2.2,5.9) \|$ & $2.9(1.7,4.9) \|$ & $2.3(1.2,4.2) \|$ \\
\hline Major depressive disorder & $2.2(1.4,3.6) \|$ & $2.3(1.4,3.9) \|$ & $1.5(0.9,2.7)$ & $0.8(0.4,1.6)$ \\
\hline GAD & $4.0(1.9,8.5) \|$ & $4.2(1.9,9.1) \|$ & $2.1(0.9,5.1)$ & $1.7(0.6,5.2)$ \\
\hline Alcohol/substance use disorder & $2.9(1.3,6.6) \|$ & $3.3(1.4,7.6) \|$ & $2.5(1.04,5.9) \|$ & $2.5(0.9,6.5)$ \\
\hline
\end{tabular}

* See Table 3 for expansion of abbreviations.

$\uparrow$ Adjusted for age, gender, race, marital status, and education.

${ }_{+}$Adjusted for age, gender, race, marital status, education, and comorbid mental disorders.

\$Adjusted for age, gender, race, marital status, education, comorbid mental disorders, and comorbid physical illnesses.

$\| \mathrm{p}<0.05$.

stance, data suggesting a familial association between major depression and allergy has been presented. ${ }^{21}$ Similarly, future studies looking at familial rates of panic attacks and respiratory disease may be informative. ${ }^{21}$ The relation noted between panic and severity of respiratory illness and panic attack suggest a bidirectional relationship is also possible. It might be that self-reported respiratory disease and panic attacks exacerbate each other when they cooccur. For example, having asthma in addition to other lung problems may be associated with dyspnea, possibly contributing to the likelihood of the onset of panic attacks. This hypothesis requires further empirical investigation.

GAD was associated with an increased likelihood of having both respiratory and lung disease, but not with having either without the other. This association may indicate that GAD is related to, or may result from, the experience of having more than one severe respiratory disease rather than a shared etiology between respiratory disease and GAD. While the relation between alcohol/substance use disorders and lung disease fails to retain statistical significance after adjusting for physical comorbidity in these analyses, the strength of this association is noteworthy. Specifically, these analyses suggest that the relation between alcohol/substance use disorders is specific to lung disease or comorbid respiratory and lung disease, and does not, in contrast, appear to be strongly associated with respiratory disease in the absence of lung disease. Furthermore, the fact that this association is no longer significant after other comorbidity is additionally controlled suggests that part of this association may be related to physical comorbidity. Severe physical illness may lead to alcohol/substance use and abuse for self-medication purposes, or alcohol/substance abuse may lead several adverse physical consequences (eg, liver disease, kidney failure, in addition to lung disease).

Limitations of this study are significant, and the results should be interpreted only within their context. First, the data on respiratory disease is selfreported. However, self-reporting is the most commonly used form of assessment of physical illness in epidemiologic studies worldwide, and it is the most frequent method for evaluation asthma and making international comparisons in prevalence rates. ${ }^{22}$ Therefore, our findings should be comparable to other epidemiologic data. Another limitation of these data are that the physical diagnosis groups used in these analyses (ie, other lung problems) are relatively nonspecific within respiratory disease. It is therefore not possible to determine the association between

Table 5-Relation Between Mental Disorders and Respiratory Disease Among Adults in the US General Population*

\begin{tabular}{lcccc}
\hline \hline \multicolumn{1}{c}{ Mental Disorders } & $\begin{array}{c}\text { Unadjusted OR } \\
(95 \% \mathrm{CI})\end{array}$ & $\begin{array}{c}\text { Adjusted OR } \\
(95 \% \mathrm{CI}) \dagger\end{array}$ & $\begin{array}{c}\text { Adjusted OR } \\
(95 \% \mathrm{CI})+\end{array}$ & $\begin{array}{c}\text { Adjusted OR } \\
(95 \% \mathrm{CI}) \S\end{array}$ \\
\hline Panic attacks & $5.5(3.0,10.2) \|$ & $5.9(3.0,11.4) \|$ & $4.0(1.9,8.2) \|$ & $4.1(1.7,9.9) \|$ \\
Major depressive disorder & $3.9(2.1,7.4) \|$ & $2.3(1.4,3.9) \|$ & $2.2(1.1,4.8) \|$ & $0.8(0.3,2.2)$ \\
GAD & $5.0(1.8,13.4) \|$ & $4.2(1.9,9.1) \|$ & $2.1(0.7,6.3)$ & $5.1(1.2,21.6) \|$ \\
Alcohol substance use disorder & $4.4(1.6,11.8) \|$ & $3.3(1.4,7.6) \|$ & $3.4(1.1,10.5) \|$ & $3.6(0.9,14.6)$ \\
\hline
\end{tabular}

* Respiratory disease includes asthma, chronic bronchitis, or emphysema and other lung disorders. See Table 3 for expansion of abbreviations. † Adjusted for age, gender, race, marital status, and education.

†Adjusted for age, gender, race, marital status, education, and comorbid mental disorders.

\$Adjusted for age, gender, race, marital status, education, comorbid mental disorders, and comorbid physical illnesses. $\| \mathrm{p}<0.05$. 
panic attacks and specific respiratory diseases (eg, asthma vs emphysema) from these data.

Future studies that examine the association between respiratory disease and mental disorders using longitudinal, epidemiologic studies with objectively measured physical health data may be helpful in improving our understanding of this link. While the association observed here between respiratory disease and panic attacks requires replication, the consistency of these findings with previous clinical and biological data supports the plausibility of a specific relationship. ${ }^{23,24}$ Respiratory disease (eg, asthma, COPD $)^{25,26}$ and panic attacks ${ }^{27,28}$ are each leading causes of disability, health-care utilization, morbidity, and mortality among youth and adults in the community. Therefore, improving our understanding of the possible relation between these two phenomena, and ultimately determining whether the treatment of one can prevent or at least improve outcomes associated with the other, may have important implications for public health.

\section{REFERENCES}

1 Dratcu L. Panic, hyperventilation and perpetuation of anxiety. Prog Neuropsychopharmacol Biol Psychiatry 2000; 24: 1069-1089

2 Kim HF, Kunik ME, Molinari VA, et al. Functional impairment in COPD patients: the impact of anxiety and depression. Psychosomatics 2000; 41:465-471

3 Limbos MM, Joyce DP, Chan CK, et al. Psychological functioning and quality of life in lung transplant candidates and recipients. Chest 2000; 118:408-416

4 Nowobilski R. Psychosomatic correlations in patients with bronchial asthma [in Polish]. Pol Arch Med Wewn 1999; 102:1063-1067

5 Kolbe J, Vamos M, Fergusson W, et al. Differential influences on asthma self-management knowledge and self-management behavior in acute severe asthma. Chest 1996; 110:1463-1468

6 Nouwen A, Freeston MH, Labbe R, et al. Psychological factors associated with emergency room visits among asthmatic patients. Behav Modif 1999; 23:217-233

7 Laszlo G, Nicholson EM, Denison J, et al. Adverse effect of previous bronchial asthma on disability in chronic airflow obstruction. Lancet 2000; 356:737-738

8 Van Peski-Oosterbaan AS, Spinhoven P, Van der Does AJ, et al. Is there a specific relationship between asthma and panic disorder? Behav Res Ther 1996; 34:333-340

9 Vila G, Nollet-Clemencon C, de Blic J, et al. Prevalence of DSM IV anxiety and affective disorders in a pediatric population of asthmatic children and adolescents. J Affect Disord 2000; 58:223-233

10 Koltek M, Wilkes TC, Atkinson M. The prevalence of posttraumatic stress disorder in an adolescent inpatient unit. Can J Psychiatry 1998; 43:64-68

11 Yellowlees PM, Kalucy RS. Psychobiological aspects of asthma and the consequent research implications. Chest 1990; 97:628-634

12 Hurwitz EL, Morgenstern H. Cross-sectional associations of asthma, hay fever, and other allergies with major depression and low-back pain among adults aged 20-39 years in the United States. Am J Epidemiol 1999; 150:1107-1116

13 Moody L, McCormick K, Williams A. Disease and symptom severity, functional status, and quality of life in chronic bronchitis and emphysema (CBE). J Behav Med 1990; 13: 297-306

14 Kessler RC, Mickelson KD, Zhao S. Patterns and correlates of self-help group membership in the United States. Soc Policy 1997; 27:27-46

15 Kessler RC, McGonagle KA, Zhao S, et al. Lifetime and 12-month prevalence of DSM-III-R psychiatric disorders in the United States: results from the National Comorbidity Survey. Arch Gen Psychiatry 1994; 51:8-19

16 Kessler RC, DuPont RL, Berglund P, et al. Impairment in pure and comorbid generalized anxiety disorder and major depression at 12 months in two national surveys. Am J Psychiatry 1999; 156:1915-1923

17 Kessler RC, Andrews G, Mroczek D, et al. The World Health Organization Composite International Diagnostic Interview Short Form (CIDI-SF). Int J Method Psychiatr Res 1998; 7:171-185

18 Busse WW. In reply [letter]. JAMA 2001; 285:882

19 Johnson JG, Cohen P, Pine DS, et al. Association between cigarette smoking and anxiety disorders during adolescence and early adulthood. JAMA 2000; 284:2348-2351

20 Grant EN, Lyttle CS, Weiss KB. The relation of socioeconomic factors and racial/ethnic differences in US asthma mortality. Am J Public Health 2000; 12:1923-1925

21 Wamboldt MZ, Hewitt JK, Schmitz S, et al. Familial association between allergic disorders and depression in adult Finnish twins. Am J Med Genet 2000; 96:146-153

22 Worldwide variation in prevalence of symptoms of asthma, allergic rhinoconjunctivitis, and atopic eczema: ISAAC. The Institutional Study of Asthma and Allergies in Childhood (ISAAC) Steering Committee. Lancet 1998; 351:1225-1232

23 Pine DS, Klein RG, Coplan JD, et al. Differential carbon dioxide sensitivity in childhood anxiety disorders and non-ill comparison group. Arch Gen Psychiatry 2000; 57:960-967

24 Pine DS, Weese-Mayer DE, Silvestri JM, et al. Anxiety and congenital central hypoventilation syndrome. Am J Psychiatry 1994; 151:864-870

25 Sterk PJ, Buist SA, Woolcock AJ, et al. The message from the World Asthma Meeting: the Working Groups of the World Asthma Meeting, held in Barcelona, Spain, December 9-13, 1998. Eur Respir J 1998; 14:1435-1453

26 Viegi G, Scognamiglio A, Baldacci S, et al. Epidemiology of chronic obstructive pulmonary disease (COPD). Respiration 2001; 68:4-19

27 Klerman GL, Weissman MM, Ouellette R, et al. Panic attacks in the community: social morbidity and health care utilization. JAMA 1991; 265:742-746

28 Kouzis AC, Eaton WW. Psychopathology and the initiation of disability payments. Psychiatr Serv 2000; 51:908-913 